\title{
Identification and Implication of One-dimensional Intergrowths in Beam-sensitive Two-dimensional MFI Zeolite Nanosheets
}

Prashant Kumar ${ }^{1}$, Michael Tsapatsis ${ }^{2}$ and K. Andre Mkhoyan ${ }^{3}$

${ }^{1}$ University of Michigan, Ann Arbor, Ann Arbor, Michigan, United States, ${ }^{2}$ John Hopkins University, Baltimore, Maryland, United States, ${ }^{3}$ University of Minnesota, Twin Cities, Minneapolis, Minnesota, United States

The zeolite MFI is a widely used catalyst and adsorbent which also holds promise as a thin film membrane for the separation of hydrocarbon isomers and other difficult to separate mixtures. The discovery of nmthick 2-dimensional (2D) MFI nanosheets has enabled methods for thin film zeolite fabrication that open new horizons for membrane science and engineering [1]. However, the crystal structure of 2D-MFI nanosheets and its relationship to separation performance remain elusive. MFI and MEL belong to the pentasil family of zeolites, which are frameworks with long chains of interconnecting five-membered SiO tetrahedral units (pentasil chains). Differences in connectivity of pentasil chains create significantly different network of channels in MFI and MEL framework (Fig 1a-b).

Using transmission electron microscopy, we find that one- to few-unit-cells wide intergrowths of the zeolite MEL exist within 2D-MFI. The MFI framework along the a-direction is broken by insertions of near-single-unit-cell domains of MEL. Because the MEL insertions are extended only along the cdirection, we call them 1-D or near 1-D-MEL (Fig. 1d). Fast Fourier transforms (FFT) of images with such MEL insertions show elongated spots along the a*-direction as compared to a typical MFI spot pattern (Fig. 1c, d). This is an indication that presence of finite MFI domains in the a-direction has a fingerprint in reciprocal space of the crystal as elongation of spots along the $\mathrm{a}^{*}$-direction. We identify the planar distribution of these 1-dimensional (1D) or near-1D-MEL domains, and show that a fraction of individual nanosheets have high (ca. $25 \%$ by volume) MEL content while the majority of nanosheets are MEL-free (Fig 1c-e).

Atomistic simulations suggest that commensurate knitting of 1D-MEL within 2D-MFI creates more rigid and highly selective pores as compared to those in pristine MFI nanosheets and permeation experiments show an unprecedented separation factor of 60 using an industrially relevant (undiluted 1 bar xylene mixture) feed. [2-3] 
a

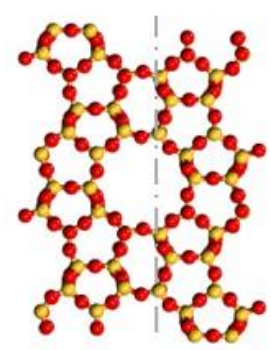

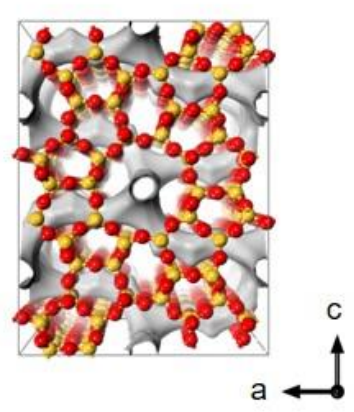

b

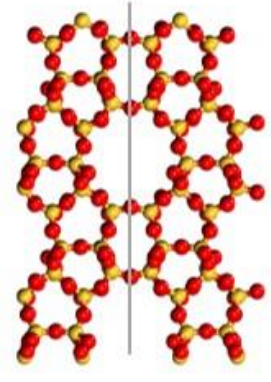

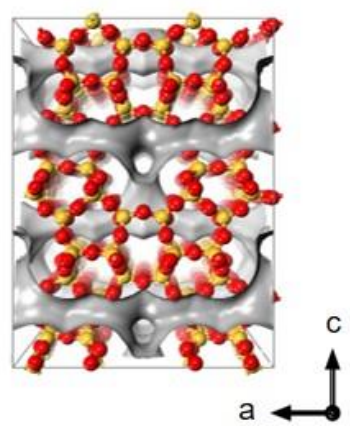

c

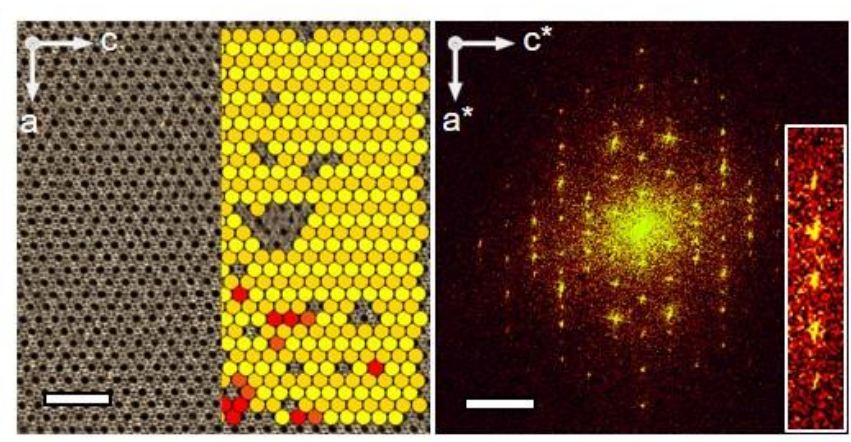

d
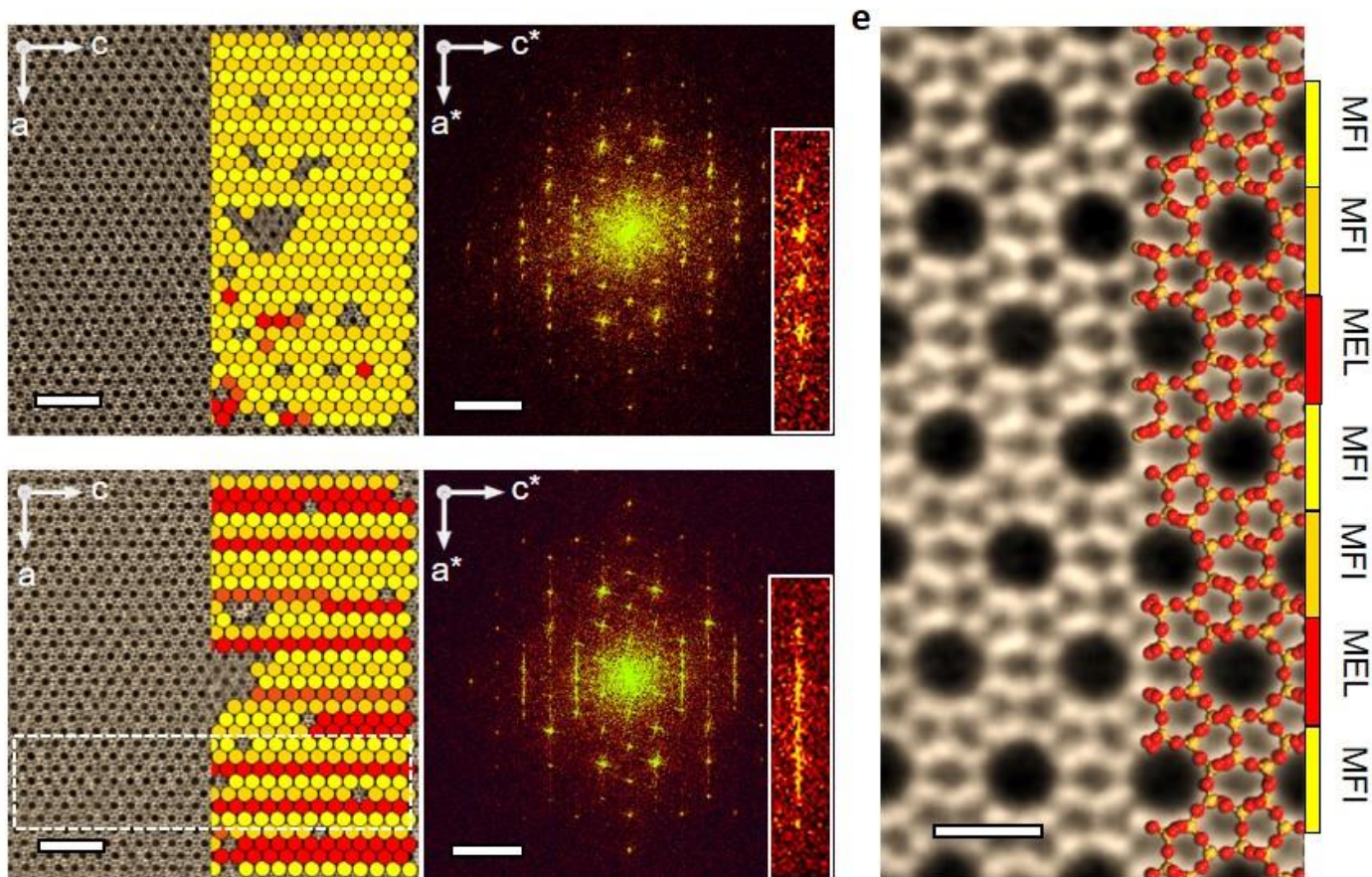

Figure 1. Figure 1. Atomic structure of nanosheet. Atomic structure (left) and the resulting porous network shown in grey (right), for a, MFI and b, MEL viewed along the b-direction that are formed by connecting pentasil chains through inversion symmetry (dashed grey line) and mirror symmetry (solid grey line), respectively. Filtered ADF-STEM image (left) and the FFT (right) for an area c, with typical MFI structure and d, with MEL domains intergrown within MFI framework. Overlaid on ADF-STEM images are colored circles for MFI (yellow) and MEL (red) unit cells that are identified using a template matching algorithm. Insets in FFT show streaking of a periodic set of spots which are an indication of finite MFI domains limited in size along the a-direction. e, Cross-correlated and averaged ADF-STEM section of MFI-MEL heterostructure from the dotted region shown in $\mathrm{d}$. The atomic structure is overlaid on top of the ADF-STEM image with perfect registration of atoms in the model with the bright dots (representing silicon atoms) in the image. Scale bars in c, $d$ are $5 \mathrm{~nm}$ for ADF-STEM image, $2 \mathrm{~nm}-1$ for FFT, and in e is $1 \mathrm{~nm}$.

\section{References}

[1] M.Y. Jeon et al, Nature. 543, (2017), 690-694.

[2] P. Kumar et al, Nat. Mat. (2020), https://doi.org/10.1038/s41563-019-0581-3.

[3] This work was primarily supported by the National Science Foundation (CBET-1705687). Parts of this work were carried out in the Characterization Facility, University of Minnesota, that receives partial support from the NSF through the MRSEC and NNIN programs (DMR-1420013). 